\title{
Neutrino Mixing and the Minimal 3-3-1 Model
}

\author{
A. Gusso ${ }^{a}$, C. A. de S. Pires ${ }^{b}$, and P. S. Rodrigues da Silva ${ }^{a}$ \\ ${ }^{a}$ Instituto de Física Teórica, Universidade Estadual Paulista, \\ Rua Pamplona 145, 01405-900 São Paulo - SP, Brazil. \\ ${ }^{b}$ Departamento de Física, Universidade Federal da Paraíba, \\ Caixa Postal 5008, 58051-970, João Pessoa - PB
}

\begin{abstract}
In the minimal 3-3-1 model charged leptons come in a non-diagonal basis. Moreover the Yukawa interactions of the model lead to a non-hermitian charged lepton mass matrix. In other words, the minimal 3-3-1 model presents a very complex lepton mixing. In view of this we check rigorously if the possible textures of the lepton mass matrices allowed by the minimal 3-3-1 model can lead or not to the neutrino mixing required by the recent experiments in neutrino oscillation.
\end{abstract}




\section{Introduction}

An alternative to the Standard Model (SM) of electro-weak interactions, the so called 3-3-1 model, was proposed some years ago [1], and one of its interesting features is that neutrino are massive from the outset. However, at the time the model was proposed there was no indication that neutrinos were massive, and in order to avoid such a mass term some fine tuning was necessary[2].

In the last few years we witnessed a dramatic change in the scenario of particle physics and the most recent experimental results, including SNO [3], K2K [4], and KamLAND [5] data, are corroborating the hypothesis of neutrino oscillation [6], which implies the existence of at least two massive neutrinos. In view of this the chief question now refers to the smallness of the neutrino masses, and in the context of 3-3-1 model, can these masses be fairly accommodated? It was shown in Ref. [7] that in fact they can. The model dispose of a type II seesaw mechanism which provides small masses to neutrinos. This could put an end point on the subject if the size of neutrinos masses were the only issue. Nevertheless, the experiments are showing not only that neutrinos have a tiny mass, but are also strongly

suggesting the pattern in which they mix [3, 4]. The proposal of this work is to check if the minimal 3-3-1 model can explain the pattern of neutrino mixing in accordance with the experiments.

There are two reasonable arguments to pursue this check. The first one concerns its absence in literature, and it is justifiable to look for consistency with experiment. The second one is related to the interplay that exists between neutrino and charged lepton mixing, that arises naturally inside the minimal 3-3-1 model. Such interplay exists because neutrinos and charged lepton masses both have the same origin: a sextet of scalars. Moreover the charged lepton mass also receive contributions of a triplet of scalars[2]. This implies that the charged leptons cannot be simply assumed to lie on a diagonal basis. Consequently, in the minimal 3-3-1 model the charged leptons are non-trivially interconnected to the neutrino physics and play some role in it, and we should be able to know better how this connection is realized under the light of the recent results on neutrino oscillation.

We organize this work as follows. First, in Sec. 2, we introduce the necessary ingredients of the model, presenting the texture of neutrino and charged lepton mass matrix of the model. Then, in Sec. 3, we examine the distinct possibilities for the lepton mixing matrices suggested by the results of neutrino oscillation. Finally, in Sec. 4, we present our conclusions.

\section{Lepton sector of the minimal 3-3-1 model}

In this section, we are going to present the ingredients necessary to obtain the mass matrix for neutrinos and charged leptons in the minimal 3-3-1 model. The gauge group under consideration is $S U_{c}(3) \otimes S U_{L}(3) \otimes U_{X}(1)$, which spontaneously breaks to the usual $S U_{c}(3) \otimes$ $S U_{L}(2) \otimes U_{Y}(1)$. In its minimal version, the scalar content that interacts with the lepton 
sector is composed by a triplet, $\eta=\left(\eta^{0}, \eta_{1}^{-}, \eta_{2}^{+}\right)^{T} \sim(\mathbf{1}, \mathbf{3}, 0)$ and a sextet $S$ [2],

$$
S=\left(\begin{array}{ccc}
\sigma_{1}^{0} & h_{1}^{-} & s_{2}^{+} \\
h_{1}^{-} & H_{1}^{--} & \sigma_{2}^{0} \\
h_{2}^{+} & \sigma_{2}^{0} & H_{2}^{++}
\end{array}\right) \sim(\mathbf{1}, \mathbf{6}, 0)
$$

where we have included their assigned transformation properties under the gauge group $S U_{c}(3) \otimes S U_{L}(3) \otimes U_{X}(1)$.

The leptons are arranged in the fundamental representation of the gauge group $S U_{L}(3)$, and for each family we have,

$$
\Psi_{a_{L}}=\left(\begin{array}{c}
\nu_{a} \\
e_{a} \\
e_{a}^{c}
\end{array}\right)_{L} \sim(\mathbf{1}, \mathbf{3}, 0)
$$

where $a=1,2,3$ labels the different families.

The Yukawa interactions that generate the lepton masses are given by,

$$
\mathcal{L}_{l}^{Y}=\frac{1}{2} G_{a b} \overline{\left(\Psi_{a L}\right)^{c}} S^{*} \Psi_{b_{L}}+\frac{1}{2} F_{a b} \epsilon^{i j k} \overline{\left(\Psi_{i a L}\right)^{c}} \Psi_{j b L} \eta_{k}^{*}+\text { H.c. }
$$

When the neutral scalars develop their respective vacuum expectation values, $\left\langle\sigma_{1}^{0}\right\rangle=v_{\sigma_{1}}$, $<\sigma_{2}^{0}>=v_{\sigma_{2}}$ and $<\eta^{0}>=v_{\eta}$, neutrinos and charged leptons acquire the following mass matrices,

$$
M_{\nu}=\left(\begin{array}{ccc}
G_{11} & G_{12} & G_{13} \\
G_{21} & G_{22} & G_{23} \\
G_{31} & G_{32} & G_{33}
\end{array}\right) v_{\sigma_{1}}
$$

and

$$
M_{l}=\left(\begin{array}{ccc}
G_{11} v_{\sigma_{2}} & \left(G_{12} v_{\sigma_{2}}+F_{12} v_{\eta}\right) & \left(G_{13} v_{\sigma_{2}}+F_{13} v_{\eta}\right) \\
\left(G_{21} v_{\sigma_{2}}-F_{12} v_{\eta}\right) & G_{22} v_{\sigma_{2}} & \left(G_{23} v_{\sigma_{2}}+F_{23} v_{\eta}\right) \\
\left(G_{31} v_{\sigma_{2}}-F_{13} v_{\eta}\right) & \left(G_{32} v_{\sigma_{2}}-F_{23} v_{\eta}\right) & G_{33} v_{\sigma_{2}}
\end{array}\right),
$$

respectively.

The question we are going to pursue next is whether it is possible to have some configuration where these interdependent matrices assume a realistic form. In other words, if there exist values of Yukawa couplings, $G_{a b}$ and $F_{a b}$, for which the expected neutrino mixing as well as the charged lepton mass spectrum can come about naturally. 
We start by noticing that lepton mass matrices in Eqs. (4) and (5), can be diagonalized as follows,

$$
M_{l}^{D}=V_{e L}^{\dagger} M_{l} V_{e R}, \quad M_{\nu}^{D}=V_{\nu}^{\dagger} M_{\nu} V_{\nu}
$$

where we have used the notation, $M^{D} \equiv \operatorname{Diag}\left(m_{1}, m_{2}, m_{3}\right)$, with $m_{i}$ being the physical lepton masses. The matrices $V$ transform the lepton fields in the interaction eigenstates into mass eigenstates and, in principle, they are different for left-handed and right-handed fields. These diagonalization matrices $V_{e L}, V_{e R}$ and $V_{\nu}$ combine themselves in the charged currents of the model which, after the $3-3-1$ breaking to the $S U(3)_{C} \otimes U(1)_{e m}$, are given by:

$$
\mathcal{L}_{l}^{C C}=-\frac{g}{\sqrt{2}}\left\{\overline{e_{L}} \gamma^{\mu} O^{W} \nu_{L} W_{\mu}^{-}+\overline{\left(e_{R}\right)^{c}} O^{V} \gamma^{\mu} \nu_{L} V_{\mu}^{+}+\overline{\left(e_{R}\right)^{c}} O^{U} \gamma^{\mu} e_{L} U_{\mu}^{++}\right\}+\text {H.c. }
$$

where we are omitting family indices and $O^{W}=V_{e L}^{T} V_{\nu}, O^{V}=V_{e R}^{T} V_{\nu}$ and $O^{U}=V_{e R}^{T} V_{e L}$ are the three mixing matrices involved in the charged currents and all of them can be chosen to be of the CKM type. Besides, we will be considering the simplest case of a zero CP violating phase, since this phase is irrelevant throughout our analysis. This means that these matrices are real, and we can work with their transpose instead of hermitian conjugate. The parameterization we assume here for the mixing matrices is:

$$
O^{W, V, U}=\left(\begin{array}{ccc}
c_{13} c_{12} & s_{12} c_{13} & s_{13} \\
-s_{12} c_{23}-s_{23} s_{13} c_{12} & c_{23} c_{12}-s_{23} s_{13} s_{12} & s_{23} c_{13} \\
s_{23} s_{12}-s_{13} c_{23} c_{12} & -s_{23} c_{12}-s_{13} s_{12} c_{23} & c_{23} c_{13}
\end{array}\right)
$$

where we have used the short form $c_{i j} \equiv \cos \theta_{i j}$ and $s_{i j} \equiv \sin \theta_{i j}$.

From the three mixing matrices that form the lepton mixing in Eq. (7), we have experimental information only upon the angles of $O^{W}$, the mixing matrix involved in the neutrino oscillation experiments. However the information we have is not enough to determine uniquely the three angles in Eq. (8). What we know is that the recent analysis of atmospheric neutrino oscillation still favor $\nu_{\mu}-\nu_{\tau}$ oscillation with an almost maximal mixing $0.92<\sin ^{2} 2 \theta_{\text {atm }} \leq 1.0$ at $90 \%$ C.L. [8]. We also have that the oscillation among $\nu_{e}-\nu_{\mu}$ is almost settled as the explanation for the solar neutrino problem. Here the recent results allow $0.25 \leq \sin ^{2} \theta_{\text {sun }} \leq 0.40$ and $0.6 \leq \cos ^{2} \theta_{\text {sum }} \leq 0.75(90 \%$ C.L. $)[9,10]$. Since the CHOOZ experiment failed to see the disappearance of $\bar{\nu}_{e}$, we also have $0 \leq \sin ^{2} 2 \theta_{\text {chz }}<0.1$ (90\% C.L.) [11].

For our proposal here we have to fix the angles $\theta_{a t m}\left(\theta_{23}\right.$ in Eq. (8)) and $\theta_{c h z}\left(\theta_{13}\right.$ in Eq. (8)). This is straightforwardly done by taking the best fit for $\theta_{\text {atm }}=45^{\circ}$, while $\theta_{\text {chz }}=0^{\circ}$, in agreement with the above presented results. The angle involved in the solar neutrino oscillation $\left(\theta_{12}\right)$ is the one that allows for a certain range of values. Fortunately, we can keep it as a free parameter. For simplicity let us take $\sin \theta_{\text {sun }}=s$ and $\cos \theta_{\text {sun }}=c$. We then are left with the so called maximal mixing pattern for $O^{W}$, according to the parameterization 
in Eq. (8):

$$
O^{W}=\left(\begin{array}{ccc}
c & s & 0 \\
\frac{-s}{\sqrt{2}} & \frac{c}{\sqrt{2}} & \frac{1}{\sqrt{2}} \\
\frac{s}{\sqrt{2}} & -\frac{c}{\sqrt{2}} & \frac{1}{\sqrt{2}}
\end{array}\right)
$$

Henceforth we will focus our attention on this particular pattern concerning the neutrino mixing.

\section{$3 \quad$ Possible lepton mixing}

According to Eq. (5), the charged lepton mass matrix is necessarily non-diagonal, compelling us to analyze only two cases. The first one is the case where neutrino mass matrix is diagonal, and the mixing in the charged current is due only to the charged lepton sector. The second possibility is to have both neutrino and charged lepton mass matrices non-diagonal, resulting in a mixing matrix given by $O^{W}=V_{e L}^{T} V_{\nu}$.

\subsection{First Case}

Let us begin our analysis by the case where neutrinos are in a diagonal mass basis. In this case, $O^{W}=V_{e L}^{T}, M_{\nu}=\operatorname{Diag}\left(G_{11} v_{\sigma_{1}}, G_{22} v_{\sigma_{1}}, G_{33} v_{\sigma_{1}}\right)$ and $M_{l}$ takes the form

$$
M_{l}=\left(\begin{array}{ccc}
G_{11} v_{\sigma_{2}} & F_{12} v_{\eta} & F_{13} v_{\eta} \\
-F_{12} v_{\eta} & G_{22} v_{\sigma_{2}} & F_{23} v_{\eta} \\
-F_{13} v_{\eta} & -F_{23} v_{\eta} & G_{33} v_{\sigma_{2}}
\end{array}\right)
$$

This texture is very peculiar since the non-diagonal elements are anti-symmetric and, as far as we know, there is no approach dealing with such a texture in literature. Now let us check if we are able to obtain the mixing in Eq. (9) taking into account the texture in Eq. (10). It is suitable to remark that when $V_{\nu}=I$ then $O^{W}=V_{e L}^{T}$ and $O^{V}=V_{e R}^{T}$, meaning that $V_{e L}^{T}$ is given solely by the maximal mixing matrix, Eq. (9).

In this case $O^{V}=V_{e R}^{T}$. With this and using the pattern of a CKM matrix in Eq. (8) for $O^{V}$, we have that

$$
V_{e R}=\left(\begin{array}{ccc}
c_{13} c_{12} & -s_{12} c_{23}-s_{23} s_{13} c_{12} & s_{23} s_{12}-s_{13} c_{23} c_{12} \\
s_{12} c_{13} & c_{23} c_{12}-s_{23} s_{13} s_{12} & -s_{23} c_{12}-s_{13} s_{12} c_{23} \\
s_{13} & s_{23} c_{13} & c_{23} c_{13}
\end{array}\right) .
$$

Starting with these assignments for the matrices $V_{e L}$ and $V_{e R}$, the charged lepton mass matrix, Eq. (10), is connected to them through $M_{l}=V_{e L} M_{l}^{D} V_{e R}^{T}$, where $M_{l}^{D}=\operatorname{Diag}\left(m_{e}, m_{\mu}, m_{\tau}\right)$. 
Since we know the charged lepton masses we can check if there exists a range of values for the mixing angles that is compatible with this equation, which we write as,

$$
V_{e L} M_{l}^{D} V_{e R}^{T}=\left(\begin{array}{ccc}
M_{11} & M_{12} & M_{13} \\
M_{21} & M_{22} & M_{23} \\
M_{31} & M_{32} & M_{33}
\end{array}\right),
$$

where we have defined,

$$
\begin{aligned}
& M_{12}=c c_{13} s_{12} m_{e}+\frac{s}{\sqrt{2}}\left[\left(s_{12} s_{13} s_{23}-c_{12} c_{23}\right) m_{\mu}-\left(c_{12} s_{23}+s_{12} s_{13} c_{23}\right) m_{\tau}\right], \\
& M_{21}=s c_{12} c_{13} m_{e}-\frac{c}{\sqrt{2}}\left[\left(s_{12} c_{23}+c_{12} s_{13} s_{23}\right) m_{\mu}+\left(s_{12} s_{23}-c_{12} s_{13} c_{23}\right) m_{\tau}\right], \\
& M_{13}=c s_{13} m_{e}+\frac{s}{\sqrt{2}}\left[-c_{13} s_{23} m_{\mu}+c_{13} c_{23} m_{\tau}\right], \\
& M_{31}=\frac{1}{\sqrt{2}}\left[-\left(s_{12} c_{23}+c_{12} s_{13} s_{23}\right) m_{\mu}+\left(s_{12} s_{23}-c_{12} s_{13} c_{23}\right) m_{\tau}\right], \\
& M_{23}=s s_{13} m_{e}+\frac{c}{\sqrt{2}}\left[c_{13} s_{23} m_{\mu}-c_{13} c_{23} m_{\tau}\right], \\
& M_{32}=\frac{1}{\sqrt{2}}\left[\left(c_{12} c_{23}-s_{12} s_{13} s_{23}\right) m_{\mu}-\left(s_{12} s_{13} c_{23}+c_{12} s_{23}\right) m_{\tau}\right], \\
& M_{11}=c c_{12} c_{13} m_{e}+\frac{s}{\sqrt{2}}\left[\left(s_{12} c_{23}+c_{12} s_{13} s_{23}\right) m_{\mu}+\left(s_{12} s_{23}-c_{12} s_{13} c_{23}\right) m_{\tau}\right], \\
& M_{22}=s s_{12} c_{13} m_{e}+\frac{c}{\sqrt{2}}\left[\left(c_{12} c_{23}-s_{12} s_{13} s_{23}\right) m_{\mu}+\left(c_{12} s_{23}+s_{12} s_{13} c_{23}\right) m_{\tau}\right], \\
& M_{33}=\frac{1}{\sqrt{2}}\left[c_{13} s_{23} m_{\mu}+c_{13} c_{23} m_{\tau}\right] .
\end{aligned}
$$

According to Eqs. (4) and (5), once we impose the neutrino mass matrix is diagonal, the texture of the charged lepton mass matrix automatically emerges and it is such that the non-diagonal elements are anti-symmetric as displayed in Eq. (10). This implies that the non-diagonal elements in Eq. (13) above have to be anti-symmetric, $M_{i j}=-M_{j i}$ for $i \neq j$. These conditions can be translated into the following set of non-linear equations for the mixing angles:

$$
\begin{aligned}
& \sqrt{2}\left[c s_{12} c_{13}+s c_{12} c_{13}\right] m_{e}+\left[s\left(s_{12} s_{13} s_{23}-c_{12} c_{23}\right)-c\left(s_{12} c_{23}+c_{12} s_{13} s_{23}\right)\right] m_{\mu} \\
& +\left[c\left(c_{12} s_{13} c_{23}-s_{12} s_{23}\right)-s\left(c_{12} s_{23}+s_{12} s_{13} c_{23}\right)\right] m_{\tau}=0, \\
& \sqrt{2} c s_{13} m_{e}-\left[s c_{13} s_{23}+s_{12} c_{23}+c_{12} s_{13} s_{23}\right] m_{\mu}+\left[s c_{13} c_{23}+s_{12} s_{23}-c_{12} s_{13} c_{23}\right] m_{\tau}=0 \\
& \sqrt{2} s s_{13} m_{e}+\left[c c_{13} s_{23}+c_{12} c_{23}-s_{12} s_{13} s_{23}\right] m_{\mu}-\left[c c_{13} c_{23}+c_{12} s_{23}+s_{12} s_{13} c_{23}\right] m_{\tau}=0 .
\end{aligned}
$$

Motivated by the fact that the charged lepton masses differ by orders of magnitude the first naive attempt we could try in order to see if this system admits a solution is to assume 
that the coefficients of each mass term vanishes. In the sequence we are going to show that no solution can be found in this way and, in this case, we have to allow for any combination of the mass coefficients to cancel each other, which will need some numerical computation.

We start by making the coefficients of $m_{e}, m_{\mu}$ and $m_{\tau}$ in Eq. (14), to vanish identically. This leads automatically to the following constraints,

$$
c s_{12}=-s c_{12}
$$

and

$$
s_{13}=0 \text {. }
$$

For simplicity, we are going to assume that $c_{13}=+1^{*}$. The constraint in Eq. (16) allows some simplification in Eq. (14) and we can extract further constraints from it, namely,

$$
\begin{aligned}
& s c_{23}=-s_{12} s_{23}, \\
& s s_{23}=-s_{12} c_{23}, \\
& c c_{23}=-c_{12} s_{23}, \\
& c s_{23}=-c_{12} c_{23} .
\end{aligned}
$$

Taking into account the constraint Eq. (15) together with these equations, it is straightforward to see that we only have a solution for this system if $s=0$ or $c=0$, which is not the case since the small angle MSW solution is already ruled out. In this way we have shown that our naive analytical approach does not allow us to obtain a conclusion about the existence of a solution.

We have then to resort to numerical calculations. The numerical analysis was done using the package for solving systems of non-linear equations described in Ref. [12]. We fixed the lepton masses as, $m_{e}=0.51 \mathrm{MeV}, m_{\mu}=105.66 \mathrm{MeV}$ and $m_{\tau}=1777.0 \mathrm{MeV}$, and assumed values for $s$ and $c$ in the range: $0.439941<s \simeq c<0.686221$ [6]. No solution for the system Eq. (14) was found in this range. This analysis is sufficient to conclude that lepton mixing in minimal 3-3-1 model does not allow for the pattern of charged lepton masses given by Eq. (10). Hence, we should look for another scenario where neutrinos and charged lepton mass matrices are both in a non-diagonal basis. That is what we will do next.

\subsection{Second Case}

Another possible scenario is one where both neutrino and charged lepton mass matrices are non-diagonal. In this situation, the mixing in Eq. (9) is generated partially by the diagonalization of neutrino mass matrix and partially by the diagonalization of charged lepton mass matrix. The only way of separating Eq. (9) in these two matrices is to have

*It can be shown that $c_{13}=-1$ would lead to the same conclusions, although to different charged lepton mass elements in Eq. (13). 
maximal mixing between $\nu_{\mu}$ and $\nu_{\tau}$ coming from the charged lepton sector and the mixing in the $\nu_{e}$ to $\nu_{\mu}$ oscillation coming from the neutrino sector. In this way we have

$$
O^{W}=V_{e L}^{T} V_{\nu}=\left(\begin{array}{ccc}
c & s & 0 \\
\frac{-s}{\sqrt{2}} & \frac{c}{\sqrt{2}} & \frac{1}{\sqrt{2}} \\
\frac{s}{\sqrt{2}} & -\frac{c}{\sqrt{2}} & \frac{1}{\sqrt{2}}
\end{array}\right)=\left(\begin{array}{ccc}
1 & 0 & 0 \\
0 & \frac{1}{\sqrt{2}} & \frac{1}{\sqrt{2}} \\
0 & -\frac{1}{\sqrt{2}} & \frac{1}{\sqrt{2}}
\end{array}\right) \times\left(\begin{array}{ccc}
c & s & 0 \\
-s & c & 0 \\
0 & 0 & 1
\end{array}\right)
$$

where,

$$
V_{e L}^{T}=\left(\begin{array}{ccc}
1 & 0 & 0 \\
0 & \frac{1}{\sqrt{2}} & \frac{1}{\sqrt{2}} \\
0 & -\frac{1}{\sqrt{2}} & \frac{1}{\sqrt{2}}
\end{array}\right)
$$

and,

$$
V_{\nu}=\left(\begin{array}{ccc}
c & s & 0 \\
-s & c & 0 \\
0 & 0 & 1
\end{array}\right)
$$

From the Lagrangian term containing the singly charged bilepton, Eq. (7), we have that $O^{V}=V_{e R}^{T} V_{\nu}$, with the diagonalization matrices given above. We then parameterize $O^{V}$ according to the usual CKM pattern,

$$
O^{V}=\left(\begin{array}{ccc}
c_{12} c_{13} & s_{12} c_{13} & s_{13} \\
-s_{12} c_{23}-c_{12} s_{13} s_{23} & c_{12} c_{23}-s_{12} s_{13} s_{23} & c_{13} s_{23} \\
s_{12} s_{23}-c_{12} s_{13} c_{23} & -c_{12} s_{23}-s_{12} s_{13} c_{23} & c_{13} c_{23}
\end{array}\right) .
$$

Dissociating it as a product of three different rotation matrices,

$$
O^{V}=\left(\begin{array}{ccc}
1 & 0 & 0 \\
0 & c_{23} & s_{23} \\
0 & -s_{23} & c_{23}
\end{array}\right) \times\left(\begin{array}{ccc}
c_{13} & 0 & s_{13} \\
0 & 1 & 0 \\
-s_{13} & 0 & c_{13}
\end{array}\right) \times\left(\begin{array}{ccc}
c_{12} & s_{12} & 0 \\
-s_{12} & c_{12} & 0 \\
0 & 0 & 1
\end{array}\right)
$$

we recognize the last matrix at the right hand side of Eq. (22) as the neutrino mixing matrix given above in Eq. (20) which implies $c_{12}=c$ and $s_{12}=s$.

After this, the appropriate form of $V_{e R}$ is then automatically obtained as follows:

$$
V_{e R}^{T}=\left(\begin{array}{ccc}
1 & 0 & 0 \\
0 & c_{23} & s_{23} \\
0 & -s_{23} & c_{23}
\end{array}\right) \times\left(\begin{array}{ccc}
c_{13} & 0 & s_{13} \\
0 & 1 & 0 \\
-s_{13} & 0 & c_{13}
\end{array}\right)=\left(\begin{array}{ccc}
c_{13} & 0 & s_{13} \\
-s_{23} s_{13} & c_{23} & s_{23} c_{13} \\
-c_{23} s_{13} & -s_{23} & c_{23} c_{13}
\end{array}\right)
$$


With $V_{e R}$ given above, Eq. (23), and $V_{\nu}$ given by Eq. (20), we are able to obtain the texture of the neutrino and charged lepton mass matrices. For the neutrinos we have:

$$
V_{\nu} M_{\nu}^{D} V_{\nu}^{T}=\left(\begin{array}{ccc}
m_{1} c^{2}+m_{2} s^{2} & \left(m_{2}-m_{1}\right) c s & 0 \\
\left(m_{2}-m_{1}\right) c s & m_{1} s^{2}+m_{2} c^{2} & 0 \\
0 & 0 & m_{3}
\end{array}\right) .
$$

When this is confronted with $M_{\nu}$ in Eq. (5) we conclude that $G_{13}=G_{23}=0$. As a consequence, we are left with a peculiar texture for the charged lepton mass matrix,

$$
M_{l}=\left(\begin{array}{ccc}
G_{11} v_{\sigma_{2}} & \left(G_{12} v_{\sigma_{2}}+F_{12} v_{\eta}\right) & F_{13} v_{\eta} \\
\left(G_{12} v_{\sigma_{2}}-F_{12} v_{\eta}\right) & G_{22} v_{\sigma_{2}} & F_{23} v_{\eta} \\
-F_{13} v_{\eta} & -F_{23} v_{\eta} & G_{33} v_{\sigma_{2}}
\end{array}\right) .
$$

We must check now if these assignments are plausible by verifying if the diagonalization matrices so obtained drive us to such a texture. We then compute $V_{e L} M_{l}^{D} V_{e R}^{T}$ where $V_{e L}$ is given by Eq. (19), and $V_{e R}$ by Eq. (23),

$$
\begin{aligned}
& M_{l}=V_{e L} M_{l}^{D} V_{e R}^{T}=\left(\begin{array}{ccc}
1 & 0 & 0 \\
0 & \frac{1}{\sqrt{2}} & -\frac{1}{\sqrt{2}} \\
0 & \frac{1}{\sqrt{2}} & \frac{1}{\sqrt{2}}
\end{array}\right) \times\left(\begin{array}{ccc}
m_{e} & 0 & 0 \\
0 & m_{\mu} & 0 \\
0 & 0 & m_{\tau}
\end{array}\right) \times\left(\begin{array}{ccc}
c_{13} & 0 & s_{13} \\
-s_{23} s_{13} & c_{23} & s_{23} c_{13} \\
-c_{23} s_{13} & -s_{23} & c_{23} c_{13}
\end{array}\right)= \\
& \left(\begin{array}{ccc}
c_{13} m_{e} & 0 & s_{13} m_{e} \\
\frac{1}{\sqrt{2}}\left(-s_{23} s_{13} m_{\mu}+c_{23} s_{13} m_{\tau}\right) & \frac{1}{\sqrt{2}}\left(c_{23} m_{\mu}+s_{23} m_{\tau}\right) & \frac{1}{\sqrt{2}}\left(s_{23} c_{13} m_{\mu}-c_{23} c_{13} m_{\tau}\right) \\
\frac{1}{\sqrt{2}}\left(-s_{23} s_{13} m_{\mu}-c_{23} s_{13} m_{\tau}\right) & \frac{1}{\sqrt{2}}\left(c_{23} m_{\mu}-s_{23} m_{\tau}\right) & \frac{1}{\sqrt{2}}\left(s_{23} c_{13} m_{\mu}+c_{23} c_{13} m_{\tau}\right)
\end{array}\right) .
\end{aligned}
$$

Let us look at the consequences of this result. We first observe that by identifying Eq. (25) with Eq. (26) we obtain the following equations,

$$
\begin{aligned}
& -s_{23} s_{13} m_{\mu}+c_{23} s_{13} m_{\tau}=0, \\
& s_{13} m_{e}=\frac{1}{\sqrt{2}}\left(s_{13} s_{23} m_{\mu}+s_{13} c_{23} m_{\tau}\right), \\
& c_{23} m_{\mu}-s_{23} m_{\tau}=-s_{23} c_{13} m_{\mu}+c_{23} c_{13} m_{\tau} .
\end{aligned}
$$

We can analyze two possibilities, namely, $s_{13}=0$ and $s_{13} \neq 0$. For the case $s_{13}=0$ (assuming $\left.c_{13}=+1\right)$, these equations have a solution for $c_{23}=-s_{23}$.

However, the fact that elements $G_{a b}$ are present in both mass matrices, of neutrinos and charged leptons, leads to tight constraints on their values. For instance, by comparing the matrix in Eq. (5) with Eq. (24), we can extract

$$
G_{11} v_{\sigma_{1}}=m_{1} c^{2}+m_{2} s^{2},
$$


and

$$
G_{33} v_{\sigma_{1}}=m_{3} .
$$

According to Eqs. (25) and (26), after substituting the obtained value for the mixing angles, $s_{13}=0 \Rightarrow c_{13}=1$ and $c_{23}=-s_{23}=1 / \sqrt{2}, G_{11}$ and $G_{33}$ are also constrained to the charged lepton masses,

$$
G_{11} v_{\sigma_{2}}=m_{e}
$$

and

$$
G_{33} v_{\sigma_{2}}=\frac{1}{2}\left(m_{\tau}-m_{\mu}\right) \simeq \frac{1}{2} m_{\tau} .
$$

But this is not possible because $m_{1}, m_{2}$ and $m_{3}$ are of the order of $\mathrm{eV}$, while $m_{e}$ is of the order of $\mathrm{MeV}$ and $m_{\tau}$ is of the order of GeV. When we divide Eq. (28) and Eq. (29) by Eq. (30) and Eq. (31), respectively, we obtain that the ratio $v_{\sigma_{1}} / v_{\sigma_{2}}$ is about $10^{-6}$ in one case and $10^{-9}$ in the other, which is an absurd. This shows that such a solution is not possible.

Suppose now that $s_{13}$ is not null. In this situation the second equation in Eq. (27) becomes,

$$
m_{e}=\frac{1}{\sqrt{2}}\left(s_{23} m_{\mu}+c_{23} m_{\tau}\right) .
$$

Since the magnitude of the charged lepton masses are very different, there is no value for the mixing angle in Eq. (32) that makes the right hand side of this equation to be equal to the electron mass. For instance, if $s_{23}\left(c_{23}\right)$ is small, $c_{23}\left(s_{23}\right)$ is close to one and we would obtain $m_{e} \sim m_{\tau}\left(m_{\mu}\right)$, an obviously false result. This analysis is general enough and we do not even need to appeal to numerical computation to conclude that the minimal 3-3-1 model requires some extension in order to accommodate the lepton mixture.

\section{Concluding Remarks}

We have investigated the possibility of conciliating the mixing in lepton sector, suggested by the neutrino oscillation hypothesis, with the minimal version of 3-3-1 model. Due to the very particular form of the anti-symmetric Yukawa coupling with the triplet, we have shown that none of the alternatives for the mass matrix textures of charged leptons and neutrinos could generate the expected pattern for neutrino mixing. Hence, if by one side the minimal 3-3-1 model naturally explains the smallness of neutrinos masses [7], by the other side it is not able to consistently accommodate the neutrino mixing. This does not mean that the model loses its attractiveness, it still addresses additional questions not embraced by the Standard Model. However, the newest observations from neutrino physics put it clear, through the results here obtained, that further extension is needed in order to keep the privileged position that the model deserves[13].

Acknowledgements. We thank Orlando Peres for valuable informations on the recent experimental limit on $\sin ^{2} \theta_{\text {sum }}$ and $\cos ^{2} \theta_{\text {sum. }}$. A.G and P.R.S thank FAPESP while C.P thank CNPq for partial support. 


\section{References}

[1] F. Pisano and V. Pleitez, Phys. Rev. D46, 410 (1992); P. H. Frampton, Phys. Rev. Lett. 69, 2889 (1992).

[2] R. Foot, O. F. Hernández, F. Pisano and V. Pleitez, Phys. Rev. D47, 4158 (1993).

[3] SNO collaboration, Q.R. Ahmad et al., Phys. Rev. Lett. 89, 011301 (2002); Phys. Rev. Lett. 89, 011302 (2002).

[4] Super-Kamiokande Collaboration (Y. Fukuda et al.), Phys. Lett. B436, 33 (1998); Phys. Rev. Lett. 81, 1158 (1998); Erratum 81, 4279 (1998); Phys. Rev. Lett. 81, 1562 (1998); Phys. Rev. Lett. 82, 1810 (1999); Super-Kamiokande collaboration, Y. Suzuki, Nucl. Phys. B91 (Proc. Suppl.), 29 (2001); Super-Kamiokande collaboration, S. Fukuda et al., Phys. Rev. Lett. 86, 5651 (2001).

[5] KamLAND Collaboration, K. Eguchi et al., Phys. Rev. Lett. 90, 021802 (2003).

[6] J. N. Bahcall, M. C. Gonzalez-Garcia and C. Pena-Garay, JHEP 0204, 007 (2002); JHEP 0302, 009 (2003).

[7] J. C. Montero, C. A. de S. Pires and V. Pleitez, Phys. Lett. B502, 167, (2001); M. B. Tully and G. C. Joshi, Phys. Rev. D64, 011301(R) (2001).

[8] G. L. Fogli, E. Lisi, A. Marrone, and D. Montanino, hep-ph/0303064.

[9] G. L. Fogli, E. Lisi, A. Marrone, D. Montanino, A. Palazzo, and A. M. Rotunno, hep$\mathrm{ph} / 0212127$.

[10] H. Nunokawa, W. J. C. Teves, R. Zukanovich Funchal, Phys. Lett. B 562, 28 (2003); P. C. de Holanda, A. Y. Smirnov, JCAP 0302, 001 (2003).

[11] CHOOZ Collaboration, M. Apollonio et al., Phys. Lett. B 420, 397 (1998).

[12] R. B. Kearfott and M. Novoa, ACM Trans. Math. Software 16, 152 (1990).

[13] For various approaches in this directions see: Y. Okamoto and M. Yasue, Phys. Lett. B 466267 (1999); T. Kitabayashi and M. Yasue, Nucl. Phys. B 60961 (2001) and references therein; J. C. Montero, C. A. de S. Pires and V. Pleitez, Phys. Rev. D65 095001 (2002); J. C. Montero, C. A. de S. Pires, and V. Pleitez, Phys. Rev. D66 113003 (2002); J. C. Montero, C. A. de S.Pires, and V. Pleitez, Phys. Rev. D65 093017 (2002). 